\title{
Manganese is a Physiologically Relevant TORC1 Activator in Yeast and Mammals
}

Raffaele Nicastro $^{3,4}$, Hélène Gaillard ${ }^{1,2,4}$, Laura Zarzuela ${ }^{1}$, Elisabet Fernández-García1,2, Mercedes Tomé ${ }^{1}$, Néstor García-Rodríguez ${ }^{1,2}$, Marie-Pierre Péli-Gulli ${ }^{3}$, Raúl V. Dúran¹, Claudio De Virgilio ${ }^{3,5}$ and Ralf Erik Wellinger ${ }^{1,2,5}$

${ }^{1}$ Centro Andaluz de Biología Molecular y Medicina Regenerativa - CABIMER, Consejo Superior de Investigaciones Científicas - Universidad de Sevilla - Universidad Pablo de Olavide, Avda. Américo Vespucio 24, 41092 Seville, Spain

${ }_{2}^{2}$ Departamento de Genética, Facultad de Biología, Universidad de Sevilla

${ }^{3}$ University of Fribourg, Department of Biology, Chemin du Musée 10, 1700 Fribourg, Switzerland

${ }^{4}$ Both authors contributed equally.

${ }^{5}$ To whom correspondence should be addressed.

\section{Highlights}

- Mn acts as a primordial activator of TORC1

- NRAMP transporters control TORC1 through cytoplasmic Mn levels

- TORC1 regulates Mn levels through feedback circuits impinging on NRAMP transporters

- Mn-mediated TORC1 activation modulates retrograde response, autophagy, and mitophagy 


\begin{abstract}
The essential biometal manganese ( $\mathrm{Mn}$ ) functions as a cofactor for several enzymatic activities that are critical for the prevention of human diseases. Whether intracellular Mn levels may also modulate signaling events has so far remained largely unexplored. The target of rapamycin complex 1 (TORC1, mTORC1 in mammals) is a conserved protein kinase complex that requires metal co-factors to phosphorylate its downstream effectors as part of a central, homeostatic process that coordinates cell growth and metabolism in response to nutrient and/or growth factor availability. Using genetic and biochemical approaches, we show here that TORC1 activity is exquisitely sensitive to stimulation by Mn both in vivo and in vitro. Mn-mediated control of TORC1 depends on Smf1 and Smf2, two members of the family of natural resistance-associated macrophage protein (NRAMP) metal ion transporters, the turnover of which is subjected to feedback control by TORC1 activity. Notably, increased Mn levels and consequent activation of TORC1 cause retrograde dysregulation and antagonize the rapamycin-induced gene expression and autophagy programs in yeast. Because Mn also activates mTORC1 signaling in aminoacid starved human cells, our data indicate that intracellular Mn levels may constitute an evolutionary conserved physiological cue that modulates eukaryotic TORC1/mTORC1 signaling. Our findings therefore reveal a hitherto elusive connection between intracellular Mn levels, mTORC1 activity, and human diseases.
\end{abstract}




\section{Introduction}

Manganese (Mn) is a vital trace element that is required for the normal activity of the brain and nervous system by acting, among other mechanisms, as an essential, divalent metal cofactor for enzymes such as the mitochondrial enzyme superoxide dismutase (Mn-SOD) ${ }^{1}$, the apical activator of the DNA damage response kinase ATM ${ }^{2}$ or the Mn-activated glutamine synthetase ${ }^{3}$. However, Mn can also become toxic when enriched in the human body ${ }^{4}$. While mitochondria have been proposed as a preferential organelle where Mn accumulates and unfolds its toxicity by increasing oxidative stress and thus mitochondrial dysfunction ${ }^{5}$, the molecular mechanisms of Mn toxicity in humans is also related to protein misfolding, endoplasmic reticulum (ER) stress, and apoptosis ${ }^{6}$. Based on these findings, Mn homeostasis, which is coordinated by a complex interplay between various metal transporters for Mn uptake and intracellular Mn distribution, represents an essential task of eukaryotic cells, although it is yet not fully understood.

Much of our knowledge on Mn transport across the plasma membrane, into mitochondria, the ER, the Golgi, endosomes, or vacuoles comes from studies in Saccharomyces cerevisiae 7-15 (outlined in Figure 1A). Typically, $\mathrm{Mn}$ is shuttled across membranes by transporters that belong to the family of natural resistance-associated macrophage proteins (NRAMP), which are highly conserved metal transporters responsible for iron (Fe) and Mn uptake ${ }^{16}$. Not surprisingly, therefore, NRAMP orthologs have also been found to cross-complement functions in yeast, mice, and humans ${ }^{17}$. One of the beststudied NRAMPs is the yeast plasma membrane protein Smf1. Interestingly, extracellular Fe or Mn supplementation triggers Bsd2 adaptor protein-dependent, Rsp5-mediated ubiquitination of Smf1, which initiates its sorting through the endocytic multivesicular body (MVB) pathway and subsequent lysosomal degradation ${ }^{18,19}$. The Smf1 paralogs Smf2 and Smf3 are less well studied, but Smf2 is predominantly localized at endosomes and its levels decrease under conditions of Mn or Fe overload 20,21. Within cells, the P-type ATPase Pmr1 (also called Bsd1) represents a key transporter that shuttles divalent calcium (Ca) and $\mathrm{Mn}$ ions into the lumen of the Golgi ${ }^{22}$. Similarly to the loss of Bsd2, which increases Mn uptake by stabilizing Smf1 at the plasma membrane, loss of Pmr1 leads to increased cytoplasmic Mn levels as a consequence of defective Mn detoxification within the Golgi. Because Mn itself functions as a superoxide dismutase, these observations also explain why 
both $b s d 2 \Delta$ and $p m r 1 \Delta$ mutants bypass the requirement for the superoxide dismutase Sod1 5,23 .

The target of rapamycin complex 1 (TORC1 in yeast, mTORC1 in mammals) is a central, highly conserved controller of cell growth and aging in eukaryotes 24. TORC1 coordinates the cellular response to multiple inputs, including nutritional availability, bioenergetic status, oxygen levels and, in multicellular organism, the presence of growth factors 25,26. In response to these diverse cues, TORC1 regulates cell growth and proliferation, metabolism, protein synthesis, autophagy, and DNA damage responses ${ }^{27,28}$. In $S$. cerevisiae, which played a pivotal role in the discovery and dissection of the TOR signaling network ${ }^{29}$, TORC1 is mainly localized on the surfaces of vacuoles and endosomes 30,31 where it integrates, among other cues, amino acid signals through the Rag GTPases and Pib2 32,33. In mammals, amino acids also activate the Rag GTPases, which then recruit mTORC1 to the lysosomal surface where it can be allosterically activated by the small GTPase Rheb that mediates the presence of growth factors and sufficient energy levels ${ }^{27,34-}$ 36. Whether TORC1/mTORC1 is also able to sample the presence of trace elements is currently not known.

In yeast, genetic evidence links elevated cytoplasmic Mn levels to TORC1 activation, since defective $\mathrm{Mn}$ detoxification in pmr1s mutants appeared to confer rapamycin resistance ${ }^{37}$. The underlying molecular mechanism(s) through which Mn may mediate rapamycin resistance, however, remains to be explored. Here, we show that enhanced Mn uptake by NRAMP transporters in $\mathrm{MnCl}_{2}$-treated cells modulates rapamycin resistance and, in turn, that TORC1 regulates NRAMP transporter stability and turnover. Moreover, intracellular Mn excess antagonizes rapamycin-induced autophagy, mitophagy, and retrograde response activation. Surprisingly, both our in vitro and in vivo analyses reveal that TORC1 protein activity is strongly activated in the presence of $\mathrm{MnCl}_{2}$ when compared to $\mathrm{MgCl}_{2}$. Together, our findings indicate that TORC1 is regulated by and regulates intracellular Mn levels and thus plays a critical role in Mn homeostasis. Importantly, our studies in human cells indicate that Mn-driven TORC1 activation is likely evolutionarily conserved and thus pinpoint new perspectives for our understanding of conditions related with Mn toxicity such as neurodegenerative disorders and aging. 


\section{Results}

\section{NRAMP transporters regulate cytoplasmic Mn levels and rapamycin resistance}

Yeast lacking the Golgi-localized P-type ATPase Pmr1, which transports divalent Ca and Mn ions from the cytoplasm into the Golgi, are resistant to the TORC1 inhibitor rapamycin suggesting that these cells have higher TORC1 activity 37,38. While the underlying mechanism remains currently elusive, proper Mn shuttling at the Golgi is key for the Golgiresident mannosyltransferase complex and for normal protein sorting, which is why cells lacking Pmr1 accumulate the general amino-acid permease Gap1 at the plasma membrane 39. Higher levels of Gap1 at the plasma membrane may in theory translate into unrestrained uptake and intracellular accumulation of amino-acids and thus hyperactivation of TORC1,

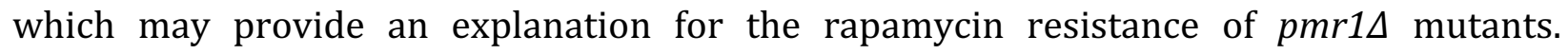
However, arguing against such a model, we found cells lacking both Pmr1 and Gap1 to remain resistant to rapamycin (Suppl. Figure 1A). We next asked whether loss of Pmr1 affects TORC1 localization. This appeared not to be the case because GFP-Tor1 localized normally to vacuolar and endosomal membranes in both exponentially growing and rapamycin-treated wild type and pmr1s cells (Suppl. Figure 1B). Moreover, loss of Pmr1 did not noticeably alter the levels of GFP-Tor1 under the same conditions (Suppl. Figure 1C). Because cells lacking Pmr1 display roughly 5-fold increased cellular levels of $\mathrm{Mn}{ }^{22}$, we then considered the possibility that Mn may have a more direct role in TORC1 activation. We therefore assessed the rapamycin-sensitivity of a $b s d 2 \Delta$ mutant that lacks the adaptor protein Bsd2, which normally mediates Rsp5-dependent degradation of both the membrane and endosomal NRAMP Mn transporters Smf1 and Smf2, respectively, when Mn or Fe ions are present in the medium (Figure $1 \mathrm{~A},{ }^{20}$ ). Interestingly, bsd2A cells were similarly sensitive to rapamycin as wild-type cells, but could, unlike wild-type cells, be rendered rapamycin resistant by the addition of $1 \mathrm{mM}$ extracellular $\mathrm{MnCl}_{2}$ (Figure $1 \mathrm{~B}$ and Suppl. Figure 2). This effect was strongly reduced in the absence of Smf2 (Figure 1C), suggesting that Smf2-dependent endosomal Mn export and consequently cytoplasmic accumulation of Mn may be required for the development of rapamycin resistance under these conditions. Further in line with such a model, we found that overexpression of the vacuolar membrane-resident Vcx1-M1 transporter, which imports Mn into the vacuolar lumen, suppressed the Mn-induced rapamycin resistance of $b s d 2 \Delta$ mutants and increased 
their sensitivity to rapamycin in the absence of extracellular $\mathrm{MnCl}_{2}$ supply (Suppl. Figure 2).

As schematically outlined in Figure 1A, several metal transporters have been associated with Mn transport in yeast. These include divalent Mn transporters, which localize at the plasma membrane (Smf1 and Pho84), endosomes (Smf2 and Atx2), the Golgi (Pmr1 and Gdt1), mitochondria (Mtm1), the vacuole (Ccc1 and Vcx1), and the ER (Spf1). To dissect which of these transporters contribute to the rapamycin resistance of pmr1 $\Delta$ cells, we monitored the growth of double mutants lacking Pmr1 and any of the corresponding metal transporters in the presence of rapamycin (Figure 1D). Interestingly, only loss of Smf1 or Smf2 reestablished rapamycin sensitivity in pmr1 $\Delta$ cells, while loss of Gdt1 or Ccc1 even slightly enhanced the rapamycin-resistance phenotype of pmr1s cells. Notably, all mutants were grown on media supplemented with $\mathrm{CaCl}_{2}$, which improved the growth of pmr1 $\Delta$ cells lacking Smf1 or Smf2 without affecting their sensitivity to rapamycin. This also indicates that $\mathrm{Ca}$ is not associated with the observed rapamycin sensitivity or resistance phenotypes (Figure 1D and Suppl. Figure 3). Taken together, our data indicate that the high intracellular Mn levels in pmr1s cells and the consequent rapamycin resistance can be reversed by either reducing Mn import through loss of Smf1, or possibly by increasing Mn sequestration in endosomes through loss of Smf2 as previously suggested ${ }^{40}$. To confirm our prediction that loss of Smf2 reduces cytoplasmic Mn levels, we also measured $\mathrm{MnCl}_{2}$ dependent telomere length shortening as an indirect proxy for cytoplasmatic and nuclear Mn levels 13. Accordingly, we found that, independently of external Ca or Mn supply, telomere length was decreased in cells lacking Pmr1 when compared to wild-type cells, increased in cells lacking Smf2, and similar between pmr1s smf2 $\Delta$ and wild-type cells (Figure 1E). Hence, loss of Smf2 suppresses the high cytoplasmic and nuclear Mn levels in

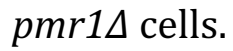

The function of metal transporters, such as Pmr1, is highly conserved across evolution as exemplified by the fact that the expression of the human Pmr1 ortholog, the secretory pathway divalent $\mathrm{Ca} / \mathrm{Mn}$ ATPase hSPCA1 (encoded by the ATP2C1 gene), can substitute for Pmr1 function in yeast 41. A similar degree of functional conservation from lower to higher eukaryotes exists for NRAMP transporters ${ }^{17}$. Because Smf2 is of specific interest in the context of the present study, we also asked whether the orthologous mouse 
proteins, i.e., the divalent metal transporter Slc11a1 and Slc11a2 isoforms, can restore rapamycin resistance in pmr1 $\operatorname{smf} 2 \Delta$ double mutants. This was indeed the case for the Slc11a2 isoform (Figure 1F), thus confirming that Pmr1 and Smf2 are evolutionarily conserved transporters needed for Mn homeostasis.

\section{Bsd2, Pmr1, and TORC1 regulate NRAMP transporter turnover}

To maintain appropriate intracellular Mn concentrations, cells adjust Smf1 and Smf2 protein levels through Bsd2-dependent and -independent, post-translational modifications that ultimately trigger their vacuolar degradation through the MVB pathway 20,23. Because our results indicated that NRAMP transporters are essential for Mn-dependent TORC1 activation, and because TORC1 is often embedded in regulatory feedback loops to ensure cellular homeostasis ${ }^{42}$, we wondered if rapamycin-mediated TORC1 inactivation may also affect Smf1 and Smf2 transporter turnover. To test this idea, we first monitored the levels of GFP-tagged Smf1 and Smf2 proteins in rapamycin-treated wild-type, pmr1 $\Delta$, and $b s d 2 \Delta$ cells. Rapamycin induced the degradation of GFP-Smf1 during a $2 \mathrm{~h}$-treatment (as measured by the increase of GFP signals on immunoblots) in wild-type, pmr1 $\Delta$, and $b s d 2 \Delta$ cells (Figure 2A and 2B), which indicates that TORC1 antagonizes the degradation of GFP-Smf1 in all three strain backgrounds. As expected, loss of Bsd2 significantly enhanced the steadystate levels of GFP-Smf1. Notably, loss of Pmr1 decreased the respective levels despite increasing the relative amount of GFP-Smf1 versus free GFP, which is indicative of a lower GFP-Smf1 degradation in these cells (Figure 2C). We therefore deem it likely that the overall lower GFP-Smf1 levels in pmr1s cells may be due to (long-term) transcriptional feedback inhibition rather than increased protein degradation. In line with these immunoblot analyses, fluorescence microscopy revealed that, in exponentially growing wild-type, $p m r 1 \Delta$, and $b s d 2 \Delta$ cells, GFP-Smf1 localized weakly, moderately, and strongly, respectively, to the plasma membrane, while rapamycin treatment induced the degradation of GFP-Smf1 as visualized by the appearance of stronger GFP signals in the vacuoles in all three mutants (Figure 2D). Thus, TORC1 promotes the expression of GFP-Smf1 at the plasma membrane and inhibits its lysosomal degradation.

In our parallel experiments, rapamycin also induced the degradation of Smf2-GFP in wild-type, $b s d 2 \Delta$ and, much less strongly, in pmr1 $\Delta$ cells (Figure $2 \mathrm{E}-\mathrm{G}$ ). Corroborating these 
findings, in exponentially growing wild-type, $b s d 2 \Delta$, and pmr1 $\Delta$ cells, Smf2-GFP localized primarily within intracellular foci that have previously been identified as part of late Golgi/endosomes ${ }^{13}$. Rapamycin treatment triggered the appearance of GFP signals in the vacuoles of wild-type and $b s d 2 \Delta$ cells, but much less in pmr1 $1 \Delta$ cells, which indicates that loss of Pmr1 may antagonize the rapamycin-mediated inactivation of TORC1 (Figure 2H). In addition, these data indicates that TORC1 inhibits the lysosomal degradation of Smf2GFP, thereby favoring its presence at endosomal membranes.

\section{Elevated levels of intracellular $\mathrm{Mn}^{2+}$ antagonize rapamycin-induced retrograde signaling, gene expression, autophagy, and mitophagy}

Growth inhibition by rapamycin mimics starvation conditions and leads to the degradation and recycling of a wide spectrum of biological macromolecules by autophagy. In this context, the pmr1 $1 \Delta$ mutant has previously been found to be defective in nutrient depletioninduced mitophagy ${ }^{43}$. We thus wondered if autophagic processing induced by rapamycin is defective in pmr1s mutants. To verify this, we took advantage of a GFP-Atg8 fusion construct to monitor autophagy through GFP-Atg8 processing and the appearance of the cleaved GFP protein in cells that had been subjected to rapamycin treatment for up to 6 hours (Figure 3A and B). Expectedly, rapamycin-induced GFP-Atg8 expression and cleavage

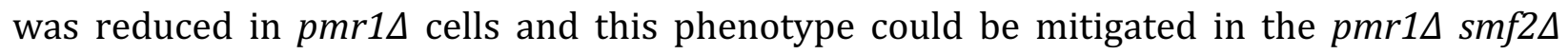
double mutant. Thus, autophagy seems to be impaired by elevated cytoplasmic Mn levels. We then wondered if this may also be the case for mitophagy, which can be assayed by following the degradation of the GFP-tagged mitochondrial membrane protein Om45 ${ }^{44}$. Rapamycin treatment led to a time-dependent up-regulation Om45-GFP protein levels in all tested strains (Figure 3C and D). However, the appearance of the cleaved GFP protein was strongly reduced in the $p m r 1 \Delta$ single mutant, while this effect was suppressed in the $p m r 1 \Delta$ smf2 $2 \Delta$ double mutant. Combined, our results suggest that the intracellular Mn flux modulates both rapamycin-induced autophagy and mitophagy.

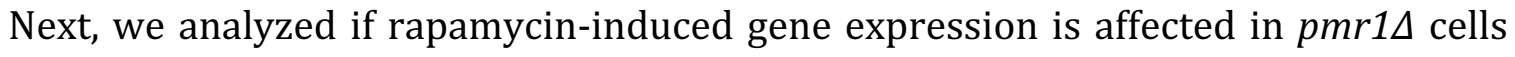
by taking advantage of a previously published microarray analysis ${ }^{13}$. This data revealed that the expression levels of genes activated by the Rtg1-3 transcription factors are reduced in pmr1s mutants (see Suppl. Table 3). Rapamycin has been shown to induce 
cytoplasmic to nuclear translocation of the Rtg1-3 transcription factors 45 and we therefore also monitored if the rapamycin-induced nuclear enrichment of a Rtg3-GFP reporter protein was affected in pmr1s mutants (Figure 3E). Indeed, nuclear Rtg3-GFP enrichment upon rapamycin treatment was impaired in cells lacking Pmr1, while nuclear Rtg3-GFP accumulation was restored in mutants lacking both Pmr1 and Smf2. Moreover, rapamycin treatment led to a strong increase in Rtg3-GFP protein levels in WT cells but not in pmr1s mutants (Figure 3F). Again, this effect was partially mitigated by combining pmr1 with smf2A. These results suggest that Mn-dependent activation of TORC1 interferes with rapamycin-dependent retrograde signaling events and thus the expression of rapamycin responsive genes.

\section{$\mathrm{MnCl}_{2}$ stimulates TORC1 kinase activity in vivo and in vitro}

So far, our results point to a direct role of Mn in TORC1 activation. Yeast Tor1 kinase was previously shown to stimulate the phosphate exchange of human PHAS-I translational repressor in the presence of $\mathrm{Mn}{ }^{46}$. We therefore determined if Mn could act as a metal cofactor for TORC1 activity in vitro using TORC1 purified from yeast cells and a truncated form of Lst4 (Lst4 ${ }^{\mathrm{Loop} ;}{ }^{47}$ ) as substrate in protein kinase assays. Intriguingly, $\mathrm{MnCl}_{2}$ not only stimulated TORC1 in vitro in a concentration-dependent manner, but also activated TORC1 much more efficiently than $\mathrm{MgCl} 2$ (with a 20-fold lower calculated $\mathrm{Km}, 43 \mu \mathrm{M}$ for $\mathrm{MnCl} 2$ versus $847 \mu \mathrm{M} \mathrm{MgCl2}$ )(Figure 4A and B). Importantly, wild-type and pmr1 $\Delta$ cells have been reported to contain $26 \mu \mathrm{M}$ and $170 \mu \mathrm{M}$ divalent $\mathrm{Mn}$ ions, respectively 48 and a similar difference $(20 \mu \mathrm{M}$ versus $160 \mu \mathrm{M})$ resulted in an 2-fold activation of TORC1 in our in vitro kinase assay. In line with these in vitro data, addition of $1 \mathrm{mM} \mathrm{MnCl}_{2}$ to the medium also significantly activated TORC1 in vivo (as assessed by the phosphorylation of the bona fide TORC1 target residue $\mathrm{Thr}^{737}$ of Sch9; ${ }^{49}$ )(Figure 4C). In addition, this effect was even observed in the absence of Gtr1, one of the subunits of the heterodimeric Rag GTPases that mediate amino acids to TORC1 within cells ${ }^{35}$. Hence, Mn-dependent activation of TORC1 does, expectedly, not run through the Rag GTPases. Nevertheless, loss of Gtr1, but not loss of Sch9 (Suppl. Figure 4A), was able to render pmr14 cells sensitive to rapamycin (Figure 4D). Similarly, loss of the proposed glutamine sensory TORC1 activator Pib2 50 or of the Fab1 complex subunits Vac7 and Vac14, which favor synthesis of phosphatidyl-inositol-3,5- 
bisphosphate that promotes membrane-tethering of TORC1 ${ }^{51}$, also rendered the pmr1S mutant sensitive to rapamycin (Suppl. Figures 4B and C). These findings suggest that under more physiological conditions, Mn-driven rapamycin resistance still requires a functional TORC1 signaling cascade.

In yeast, Mn-induced Sch9 phosphorylation is EGO-complex independent and pmr1A sch9 4 double mutants displayed a rapamycin resistant phenotype. Yet, we find that Mndriven rapamycin resistance in yeast requires TORC1 and a functional TORC1 signaling cascade including the EGO complex ${ }^{36}$ or proficient phosphatidylinositol $(3,5)$ P2 synthesis 51. These findings suggest that like in yeast, Mn-driven mTORC1 activation in human cells still depends on the EGO/Ragulator complex for proficient mTORC1 signaling.

\section{Mn activates mTORC1 signaling in human cells}

To assess whether the response of TORC1 to $\mathrm{Mn}$ is a conserved mechanism in eukaryotes, we investigated if externally supplied $\mathrm{MnCl}_{2}$ activates mTORC1 signaling in mammalian cells. For this purpose, we used the human cell lines U2OS and HEK29 that are widely used for the cellular dissection of mTORC1-mediated mechanisms in response to nutritional inputs ${ }^{52}$. First, we examined the sufficiency of Mn to activate the mTORC1 pathway by treating amino acid-starved U2OS cells and HEK293 cells with $\mathrm{MnCl}_{2}$. U2OS cells were incubated in amino acid starving medium in the presence of increasing amounts of $\mathrm{MnCl}_{2}$ (0-1 mM) for $2 \mathrm{~h}$. Then, we analyzed mTORC1 activation by assessing the phosphorylation of the mTORC1-downstream targets S6K (phospho-T389) and S6 (phospho-S235-236). As shown in Figure 5A, and as expected, both S6K and S6 were fully phosphorylated at the specific residues in the positive control (cells incubated in the presence of amino acids, $+\mathrm{AA}$ ), and completely de-phosphorylated in the negative condition (cells incubated in the absence of amino acids, with no $\mathrm{MnCl}_{2}$ ). In agreement with a positive action of $\mathrm{Mn}$ towards mTORC1 in human cells, we also observed a robust and dose-dependent increase in S6K phosphorylation and S6 phosphorylation in cells incubated in the presence of $\mathrm{MnCl}_{2}$ at concentrations higher than $0.05 \mathrm{mM}$, with a maximum phosphorylation observed at 0.5 $\mathrm{mM}$ of $\mathrm{MnCl}_{2}$. Confirming this result, we observed a similar response of S6K and S6 phosphorylation to $\mathrm{MnCl}_{2}$ treatment in HEK293 cells, with the difference that in these cells the maximum response was reached at $1 \mathrm{mM}$ (Figure 5B). These results strongly suggests 
that $\mathrm{Mn}$ is sufficient to activate the mTORC1 pathway in the absence of amino acid inputs, at least at short times (2h). To corroborate this conclusion, we tested the phosphorylation of additional downstream targets of mTORC1 in response to one fixed concentration of $\mathrm{MnCl}_{2}(0.35 \mathrm{mM})$ in U2OS cells. Confirming what we previously observed, the treatment of amino acid-starved U2OS cells with $\mathrm{MnCl}_{2}$ was sufficient to induce an increase in the phosphorylation of the downstream targets of mTORC1 S6K, S6, 4EBP1, and ULK1 (Figure 5C). Mn has been shown to activate the mTORC1 upstream kinase AKT1 ${ }^{53}$ and we find that $\mathrm{MnCl}_{2}$ also induced an increase in AKT phosphorylation in amino-acid starved cells. However, AKT phosphorylation appeared to be much less pronounced than the phosphorylation of the mTORC1 downstream targets, thus validating the specificity of Mn towards mTORC1.

Finally, to assess the physiological relevance of mTORC1 activation in response to Mn similarly as in yeast (Figure 3), we analyzed the effect of $\mathrm{MnCl}_{2}$ treatment in autophagy, a cellular process inhibited by TORC1 both in yeast and mammalian cells 54,55. For this purpose, we analyzed the autophagic marker LC3I/II 56. During autophagy initiation, LC3 is lipidated, increasing the levels of LC3II levels. The withdrawal of amino acids lead to a rapid increase in LC3 levels, as previously reported, indicating an increase in autophagy initiation (Figure 5D). Of note, the addition of $\mathrm{MnCl}_{2}$ completely abolished the increase in LC3II levels, thus confirming that Mn prevented autophagy initiation downstream of mTORC1. These results were also observed in cells incubated in the presence of chloroquine (CQ), an inhibitor of the fusion of autophagosomes with the lysosome, thus confirming that Mn influences autophagy initiation, the process controlled by mTORC1 (Figure 5E). To further corroborate this result, we also analyzed the aggregation of LC3 upon autophagosome formation in U20S cells stably expressing a GFP-LC3 construct. GFP aggregation indicates autophagosome formation in these cells. Similarly to what we observed with endogenous LC3, GFP aggregation was increased during amino acid withdrawal, but this increase was completely abolished by $\mathrm{MnCl}_{2}$ treatment (Figure $5 \mathrm{~F}-\mathrm{G}$ ). This result further confirms that the Mn-dependent activation of mTORC1 in human cells is physiologically relevant for autophagy inhibition. Altogether, our results show that Mn is sufficient to activate mTORC1 in the absence of other inputs in human cells and to inhibit 
autophagy downstream of mTORC1. These findings indicate that Mn-mediated activation of TORC1 is an evolutionarily conserved process from yeast to human.

\section{Discussion}

Studies with yeast lacking the divalent $\mathrm{Ca} / \mathrm{Mn}$ ion Golgi transporter Pmr1, which displays an increase in intracellular Mn levels due to impaired Mn detoxification ${ }^{48}$, have pinpointed a functional link between Mn homeostasis and TOR signaling 37,38. However, the underlying mechanism(s) of how Mn impinges on TORC1 has so far remained elusive. Our study identifies Mn as a metal cofactor that stimulates the enzymatic activity of the TORC1 complex both in vitro and in vivo in yeast as well as in mammalian cells. Our results support a model in which the PI3K-related protein kinase Tor1 is activated by $\mathrm{Mg}$, but requires $\mathrm{Mn}$ as a metal co-factor for maximal activity (Figure 5H). The identification of the putative Mn binding sites within TORC1 and the underlying mechanism by which Mn promotes TORC1 activity, however, will require future detailed structural and/or molecular dynamics simulation analyses.

Our current study highlights a need for coordinated control of TORC1 activity and cytoplasmic Mn levels. The latter critically depend on a set of conserved NRAMP transporters, such as yeast Pmr1 and Smf1/2 and their mammalian homologs SPCA1 41 and Slc11a2/DCT1 (57 and this work), respectively. Therapeutic compounds that affect Slc11a2/DCT1 activity, levels or turnover or that decrease in intracellular Mn levels by other means 53 should therefore have a wide spread effect on Mn-dependent enzyme activity and cell viability in mammalian cells. Accordingly, cytoplasmic Mn levels in yeast can be increased by loss of Pmr1, which causes a defect in sequestration of Mn in the Golgi. Alternatively, they can also be boosted by increased Smf1-mediated Mn uptake combined with Smf2-mediated Mn depletion in endosomes following loss of Bsd2, an adaptor protein for the NEDD4 family E3 ubiquitin ligase Rsp5 20 that normally favors vacuolar degradation of Smf1/2 9,58. In both cases, loss of Pmr1 or of Bsd2 (specifically upon addition of Mn in the medium), enhanced cytoplasmic Mn levels mediate higher TORC1 activity and hence resistance of cells to low doses of rapamycin. Conversely, TORC1 also favors the expression of Smf1 and Smf2 at the plasma membrane and at endosomes, respectively, which indicates the existence of a positive feedback control mechanism between TORC1 activity and 
cytoplasmic Mn levels. While this may ensure optimal TORC1 activity under nutrient-rich conditions, such a mechanism may perhaps also allow cells to more rapidly inactivate TORC1 when nutrients such as amino acids become limiting. Interestingly, in this context, $\mathrm{Mn}$ is also known to act as cofactor for glutamine synthetase ${ }^{3}$ that produces the TORC1stimulating amino acid glutamine ${ }^{59}$. Hence, Mn homeostasis, amino acid metabolism, and TORC1 may be subjected to multilayered feedback regulatory circuits.

Previous work has shown that chronic exposure to high levels of $\mathrm{Mn}$ causes autophagic dysfunction and hence accumulation of compromised mitochondria in mammalian astrocytes due to reduced nuclear localization of TFEB (transcription factor EB), a key transcription factor that coordinates expression of genes involved in autophagy 60. Because mTORC1 inhibits TFEB 61 and mitochondrial quality can be improved by rapamycin-induced TFEB induction (and consequent stimulation of autophagy) 62 , our study now provides a simple rationale for the observed accumulation of damaged mitochondria upon Mn exposure. Accordingly, excess Mn antagonizes autophagy and mitophagy at least in part through TORC1 activation and subsequent TFEB inhibition, which prevents proper disposal of hazardous and ROS-producing mitochondria. This model also matches well with our previous observation that rapamycin restricts cell death associated with anomalous mTORC1 hyperactivation 55. Finally, our data indicate that Mndriven TORC1 activation and the ensuing inhibition of auto- and mitophagy are also employed by yeast cells, which highlights the primordial nature of these processes.

Recent studies have shown that $\mathrm{Mn}$ is indispensable for the host defense against cytosolic, viral double-stranded DNA as it mediates activation of the DNA sensor cGAS and its downstream adaptor protein STING ${ }^{63}$. Since Mn stimulates the innate immune sensing of tumors, Mn administration has been suggested to provide an anti-tumoral effect and to improve the treatment of cancer patients ${ }^{64}$. Nevertheless, mTOR hyperactivation is known to promote tumor progression ${ }^{65}$, and carcinoma and melanoma formation have previously been associated with mutations in the human PMR1 ortholog ATP2C1 that cause HaileyHailey disease 66. Based on our findings presented here, it is therefore tempting to speculate that tumor formation in these patients is causally linked to Mn-dependent DNA replication defects ${ }^{67}$, stress response ${ }^{68}$, and mTORC1 activation. 
Exposure to Mn dust or Mn containing smoke, as a byproduct of metal welding, is well known to cause a parkinsonial-like syndrome named manganism, a toxic condition of Mn poisoning with dyskinesia ${ }^{69}$. Interestingly, while dyskinesia has been connected to Ldopamine-mediated activation of mTORC1 ${ }^{70}$, our findings suggest that Mn-driven mTORC1 hyperactivation may impair autophagy and thereby contribute to neurological diseases ${ }^{71}$. In line with this reasoning, compounds that inhibit TORC1 activity, and thus stimulate autophagy, have been suggested as therapeutics for the treatment of Parkinson's-like neurological symptoms ${ }^{72}$. In this context, Huntington disease (HD) is another example of patients suffering from a proteinopathy characterized by parkinsonial-like neurological syndromes 73. In HD patients, expansion of the polyglutamine (polyQ) tract in the $\mathrm{N}$ terminus of the huntingtin (HTT) protein leads to protein aggregation ${ }^{74}$ and, intriguingly, HD patients exhibit reduced Mn levels in the brain ${ }^{75}$. This begs the question whether the respective cells aim to evade Mn-driven mTORC1 activation, e.g. by reducing Mn uptake or sequestration of $\mathrm{Mn}$ within organelles, to stimulate HTT protein degradation via autophagy. Finally, it should be mentioned that Mn also contributes to prion formation in yeast 76 , and elevated Mn levels have been detected in blood and central nerve system of Creutzfeldt-Jakob patients ${ }^{77}$. It will therefore be exciting to further study the cell typespecific impact of Mn-driven TORC1 activation on metabolism, genome stability, checkpoint signaling, and the immune response, all processes that play a key role in neurological diseases in aging-related processes. 


\section{Materials and Methods}

\section{Reagents and antibodies}

Mouse anti-GFP (JL-8; dilution 1:5000) and rabbit anti-G-6-PDH (A9521; dilution 1:5000) were obtained from Clontech and Sigma-Aldrich, respectively. Rabbit anti-Sch9-pThr737 (dilution 1:10000) and goat anti-Sch9 (dilution 1:1000) are custom antibodies from C. De Virgilio. Peroxidase conjugated secondary antibodies goat anti-mouse (A4416) and goat anti-rabbit (A0545) were obtained from Sigma-Aldrich. Antibodies against S6 (\#2217, dilution 1:1000), phospho-S6 (Ser235/236) (\#4856, dilution 1:1000), S6K (\#2708, dilution 1:1000), phospho-S6K(T389) (\#9205, dilution 1:1000), 4EBP1 (\#9452, dilution 1:1000), phospho-4EBP1(T37/46) (\#2855, dilution 1:1000), phospho-4EBP1(Ser65) (\#9451, dilution 1:1000), AKT (\#4691, dilution 1:1000), phospho-AKT(Ser473) (\#4060, dilution 1:1000), phospho-AKT(Thr308) (\#13038, dilution 1:1000), ULK1 (ULK kit \#8359, dilution 1:1000), phospho-ULK1 (ULK kit \#8359, dilution 1:1000), LC3 AB (\#12741, dilution 1:1000), $\beta$-actin (\#4967, dilution 1:1000), mTOR (\#2983, dilution 1:200) were obtained from Cell Signalling Technology. Antibody against CD63 (SAB4700215, dilution 1:1000) was obtained from Sigma-Aldrich. Secondary antibodies donkey anti-rabbit Alexa Fluor 488 (\#R37118) and donkey anti-mouse Alexa Fluor 555 (\#A31570) were obtained from ThermoFisher. Rapamycin was purchased from Carbosynth; paraformaldehyde and chloroquine were obtained from Sigma-Aldrich.

\section{Yeast strains, Plasmids and Growth Conditions.}

Yeast strains and plasmids used in this study are listed in Supplementary Tables S1 and S2. Gene disruption and tagging were performed with standard high-efficiency recombination methods. Yeast cells were grown to mid-log phase in synthetic-complete medium (SC; $0.17 \%$ yeast nitrogen base, $0.5 \%$ ammonium sulfate, $2 \%$ glucose and appropriate aminoacids) at $30^{\circ} \mathrm{C}$. To induce autophagy and/or mitophagy, cells were treated with 200 $\mathrm{ng} / \mathrm{ml}$ rapamycin for the indicated time.

\section{Yeast Cell Lysate Preparation and Immunoblotting.}

Cells in mid-log phase were treated with $6.7 \%$ trichloroacetic acid (final concentration), pelleted, washed with 99\% acetone, dried, dissolved in urea buffer (6 M urea, $50 \mathrm{mM}$ Tris- 
$\mathrm{HCl} \mathrm{pH}$ 7.5, 1\% SDS, $1 \mathrm{mM}$ PMSF and $10 \mathrm{mM} \mathrm{NaF}$ ) and disrupted with glass beads using a Yasui Kikai homogenizer. Cell lysates were heated at $65^{\circ} \mathrm{C}$ for $10 \mathrm{~min}$ in Laemmli SDS sample buffer, centrifugated at $15^{\prime} 000 \mathrm{~g}$ for $1 \mathrm{~min}$ and the supernatants subjected to SDSPAGE and immunoblotted. GFP-Tor1, Atg8-GFP, Om45-GFP, GFP-Smf1 and Smf2-GFP were detected using a mouse anti-GFP antibody (JL-8, Clontech) and G-6-PDH was detected using a rabbit polyclonal antibody (A9521, Sigma). Quimiluminescence signals were captured in an Amersham ImageQuant 800 Imager and quantified with ImageQuant TL software (Cytiva).

\section{Drug Sensitivity Assays}

Yeast cells were adjusted in concentration to an initial A600 of 0.2, then serially diluted 1:10 and spotted onto plates without or with different drugs at the indicated concentrations (see figure legends). $\mathrm{MnCl} 2$ was added when indicated. Plates were then incubated at $30^{\circ} \mathrm{C}$ for 3 to 4 days.

\section{Analysis of Telomere Length}

Genomic DNA was isolated from yeast strains grown inYPADfor 3 days with or without the addition of $10 \mathrm{mM} \mathrm{CaCl2}$. DNA was digested with XhoI, separated on a 1\% agarose-Tris borate EDTA gel, transferred to a Hybond XL (Amersham Biosciences) membrane, and hybridized with a 32P-labeled DNA probe specific for the terminal Y' telomere fragment. The probe was generated by random hexanucleotide-primed DNA synthesis using a short Y' specific DNA template, which was generated by PCR from genomic yeast DNA using the primers $Y$ up (5'-TGCCGTGCAACAAACACTAAATCAA-3) and Y' low (5'CGCTCGAGAAAGTTGGAGTTTTTCA-3'). Three independent colonies of each strain were analyzed to ensure reproducibility.

\section{In vivo TORC1 activity assay}

In vivo TORC1 activity was assayed as previously described ${ }^{78}$. Briefly, $10 \mathrm{ml}$ of cell culture were mixed with TCA (trichloroacetic acid) at a final concentration of 6\%. After centrifugation, the pellet was washed with cold acetone and dried in a speed-vac. The pellet was resuspended in lysis buffer (50 mM Tris-HCl [pH 7.5], 5 mM EDTA, 6 M urea, 1\% SDS), 
the amount being proportional to the 0D600nm of the original cell culture. Proteins were extracted by agitation in a Precellys machine after the addition of glass beads. Subsequently, an amount of 2X Laemmli buffer (350 mM Tris-HCl [pH 6.8], 30\% glycerol, $600 \mathrm{mM}$ DTT, 10\% SDS, BBF) identical to the lysis buffer was added to the whole-cell extract and the mix was boiled at $98^{\circ} \mathrm{C}$ for 5 minutes. The analysis was carried out by SDSPAGE using phosphospecific anti-Sch9-pThr737 and anti-Sch9 antibodies.

\section{In vitro TORC1 kinase assays}

TORC1 was purified and kinase assays were performed as previously described 47 with minor modifications. For the kinase assays in the presence of various concentrations of magnesium or manganese, reactions were performed in a total volume of $30 \mu$ with kinase buffer (50 mM HEPES/NaOH [pH 7.5], $150 \mathrm{mM} \mathrm{NaCl),} 400$ ng of purified His 6 -Lst4Loop, 60 ng TORC1 (quantified with respect to the Tor1 subunit) and 640, 320, 160, 80, 40 or $20 \mu \mathrm{M}$ $\mathrm{MgCl} 2 / \mathrm{MnCl} 2$. Reaction was started by adding $2 \mu \mathrm{l}$ of ATP mix (18 mM ATP, $3.3 \mathrm{mCi}\left[\gamma^{-}\right.$ 32P]-ATP [Hartmann Analytic, SRP-501]). After addition of sample buffer, proteins were separated by SDS-PAGE, stained with SYPRO Ruby (Sigma) (loading control), and analyzed using a phosphoimager (Typhoon FLA 9500; GE Healthcare). Band intensities were quantified with ImageJ and data were analyzed with GraphPad Prism using the MichaelisMenten non-linear fitting.

\section{Cell culture}

U20S and HEK293T cell lines were obtained from the American Type Culture Collection (ATCC). GFP-LC3 expressing U20S cells were kindly provided by Dr. Eyal Gottlieb (Cancer research UK, Glasgow, UK). All the cell lines were grown in high glucose DMEM base medium (D6546 Sigma-Aldrich) supplemented with 10\% of fetal bovine serum, glutamine (2mM), penicillin (Sigma, $100 \mu \mathrm{g} \mathrm{ml-1)}$ and streptomycin (100 $\mu \mathrm{g} \mathrm{ml}-1)$, at 37으, 5\% CO2 in humidified atmosphere. All cell lines were regularly tested for mycoplasma contamination by PCR. Amino acid starvation was performed with EBSS medium (E2888 Sigma-Aldrich) supplemented with glucose at a final concentration of $4.5 \mathrm{~g} \mathrm{~L}-1$. When indicated, starvation medium was complemented with $\mathrm{MnCl} 2$ to a final concentration of $0.05-1 \mathrm{mM}$, chloroquine 
$(10 \mu \mathrm{M})$ and with amino acids, by adding MEM Amino Acids (M5550 Sigma-Aldrich), plus MEM Non-essential Amino Acid Solution (M7145 Sigma-Aldrich) and glutamine (2 mM).

\section{Confocal and fluoresence microscopy}

Yeast cells: For imaging of GFP-TOR1, GFP-Smf1 and Smf2-GFP, cells were grown exponentially and treated or not with $200 \mathrm{ng} / \mathrm{ml}$ Rapamycin for 2 hours. Images of live fluorescent were captured with an inverted spinning disk confocal microscope (Nikon Ti-E , VisiScope CSU-W1, Puchheim, Germany) that was equipped with an Evolve 512 EM-CDD camera (Photometrics), and a 10031.3 NA oil immersion Nikon CFI series objective (Egg, Switzerland). Images were then processed using the FIJI-Image software. For imaging of Rtg3-GFP, cells were grown at $26^{\circ} \mathrm{C}$ in SD-ura minimal medium (monosodium glutamate is used as a nitrogen source) to exponential phase and treated or not with $200 \mathrm{ng} / \mathrm{ml}$ rapamycin for $30 \mathrm{~min}$. Images were obtained by projection of a focal plane image derived from wide-field fluorescence microscopy (DM-6000B, Leica) at 100x magnification using L5 filters and a digital chargecoupled device camera (DFC350, Leica). Pictures were processed with LAS AF (Leica).

Human cells: $8 \times 10^{5}$ cells were grown in coverslips for $24 \mathrm{~h}$ and treated for $4 \mathrm{~h}$ as indicated. Thereafter, cells were fixed with 4\% paraformaldehyde in PBS for 10 mins at room temperature. For GFP-LC3 assessment, after three washes with PBS, coverslips were mounted with Prolong containing DAPI (Invitrogen). Samples were imaged with a Zeiss Apotome microscope. GFP aggregation in microscopy images was assessed using ImageJ software. For immunostaining, after fixation, cells were permeabilized 10 minutes using Triton-X100 0.05\% and unspecific sites were blocked in BSA 5\%-PBS for 30 minutes on a rocking platform at room temperature. Incubation with the primary antibodies against mTORC1 and CD63 was carried out at room temperature for one hour. Cells were then incubated with the secondary antibody for another hour at room temperature. Finally, the cover slips were mounted on slides with Prolong containing DAPI (Invitrogen) and the samples were imaged with a LEICA confocal SP4 microscope. 


\section{Acknowledgments}

We thank María Díaz de la Loza for scientific illustration work, and V. Albanèse, E. de Nadal, V. Goder, K. D. Hirschi and C. Ungermann for plasmids and strains. Research was funded by grants from the University of Seville (2020/00001326), Junta de Andalucía/European Union Regional Funds (P20-RT-01220) and EMBO (STF-8685) to R.E.W.; the Swiss National Science Foundation (310030_166474/184671) to C.D.V.; the Spanish Ministry of Science, Innovation and Universities (PGC2018-096244-B-I00) to R.D. Author L.Z. was the recipient of a predoctoral grant from the Spanish Ministry of Science, Innovation and Universities (FPU19/04914).

\section{References}

1. Weisiger, R.A., and Fridovich, I. (1973). Mitochondrial Superoxide Dismutase. J. Biol. Chem. 248.

2. Chan, D.W., Son, S.-C., Block, W., Ye, R., Khanna, K.K., Wold, M.S., Douglas, P., Goodarzi, A.A., Pelley, J., Taya, Y., et al. (2000). Purification and Characterization of ATM from Human Placenta. J. Biol. Chem. 275, 7803-7810.

3. Wedler, F.C., and Denman, R.B. (1984). Glutamine synthetase: the major Mn(II) enzyme in mammalian brain. Curr. Top. Cell. Regul. 24, 153-169.

4. COUPER, and J. (1837). On the effects of black oxide of manganese when inhaled into the lungs. Br Ann Med Pharm. 1,41-42.

5. Aguirre, J.D., and Culotta, V.C. (2012). Battles with Iron: Manganese in Oxidative Stress Protection. J. Biol. Chem. 287.

6. Harischandra, D.S., Ghaisas, S., Zenitsky, G., Jin, H., Kanthasamy, A., Anantharam, V., and Kanthasamy, A.G. (2019). Manganese-Induced Neurotoxicity: New Insights Into the Triad of Protein Misfolding, Mitochondrial Impairment, and Neuroinflammation. Front. Neurosci. 13.

7. Jensen, L.T., Ajua-Alemanji, M., and Culotta, V.C. (2003). The Saccharomyces cerevisiae High Affinity Phosphate Transporter Encoded by PH084 Also Functions in Manganese Homeostasis. J. Biol. Chem. 278.

8. Luk, E., Carroll, M., Baker, M., and Culotta, V.C. (2003). Manganese activation of superoxide dismutase 2 in Saccharomyces cerevisiae requires MTM1, a member of 
the mitochondrial carrier family. Proc. Natl. Acad. Sci. 100.

9. Portnoy, M.E., Liu, X.F., and Culotta, V.C. (2000). Saccharomyces cerevisiae Expresses Three Functionally Distinct Homologues of the Nramp Family of Metal Transporters. Mol. Cell. Biol. 20.

10. Antebi, A., and Fink, G.R. (1992). The yeast Ca(2+)-ATPase homologue, PMR1, is required for normal Golgi function and localizes in a novel Golgi-like distribution. Mol. Biol. Cell 3.

11. Thines, L., Deschamps, A., Sengottaiyan, P., Savel, O., Stribny, J., and Morsomme, P. (2018). The yeast protein Gdt1p transports Mn2+ ions and thereby regulates manganese homeostasis in the Golgi. J. Biol. Chem. 293.

12. Lapinskas, P.J., Lin, S.-J., and Culotta, V.C. (1996). The role of the Saccharomyces cerevisiae CCC1 gene in the homeostasis of manganese ions. Mol. Microbiol. 21.

13. García-Rodríguez, N., Manzano-López, J., Muñoz-Bravo, M., Fernández-García, E., Muñiz, M., and Wellinger, R.E. (2015). Manganese redistribution by calciumstimulated vesicle trafficking bypasses the need for P-type ATPase function. J. Biol. Chem. 290.

14. Gitler, A.D., Chesi, A., Geddie, M.L., Strathearn, K.E., Hamamichi, S., Hill, K.J., Caldwell, K.A., Caldwell, G.A., Cooper, A.A., Rochet, J.-C., et al. (2009). $\alpha$-Synuclein is part of a diverse and highly conserved interaction network that includes PARK9 and manganese toxicity. Nat. Genet. 41.

15. Cohen, Y., Megyeri, M., Chen, O.C.W., Condomitti, G., Riezman, I., Loizides-Mangold, U., Abdul-Sada, A., Rimon, N., Riezman, H., Platt, F.M., et al. (2013). The Yeast P5 Type ATPase, Spf1, Regulates Manganese Transport into the Endoplasmic Reticulum. PLoS One 8 .

16. Supek, F., Supekova, L., Nelson, H., and Nelson, N. (1996). A yeast manganese transporter related to the macrophage protein involved in conferring resistance to mycobacteria. Proc. Natl. Acad. Sci. 93.

17. Sacher, A., Cohen, A., and Nelson, N. (2000). Metal ion transporters from yeast to human diseases. Comp. Biochem. Physiol. Part A Mol. Integr. Physiol. 126.

18. Liu, X.F., and Culotta, V.C. (1999). Post-translation Control of Nramp Metal Transport in Yeast. J. Biol. Chem. 274. 
19. Eguez, L., Chung, Y.-S., Kuchibhatla, A., Paidhungat, M., and Garrett, S. (2004). Yeast Mn ${ }^{2+}$ Transporter, Smf1p, Is Regulated by Ubiquitin-Dependent Vacuolar Protein Sorting. Genetics 167.

20. Liu, X.F., Supek, F., Nelson, N., and Culotta, V.C. (1997). Negative Control of Heavy Metal Uptake by the Saccharomyces cerevisiae BSD2 Gene. J. Biol. Chem. 272.

21. Moreno-Cermeño, A., Obis, È., Bellí, G., Cabiscol, E., Ros, J., and Tamarit, J. (2010). Frataxin Depletion in Yeast Triggers Up-regulation of Iron Transport Systems before Affecting Iron-Sulfur Enzyme Activities. J. Biol. Chem. 285.

22. Lapinskas, P.J., Cunningham, K.W., Liu, X.F., Fink, G.R., and Culotta, V.C. (1995). Mutations in PMR1 suppress oxidative damage in yeast cells lacking superoxide dismutase. Mol. Cell. Biol. 15.

23. Liu, X.F., and Culotta, V.C. (1994). The requirement for yeast superoxide dismutase is bypassed through mutations in BSD2, a novel metal homeostasis gene. Mol. Cell. Biol. 14.

24. González, A., and Hall, M.N. (2017). Nutrient sensing and <scp $>$ TOR</scp $>$ signaling in yeast and mammals. EMBO J. 36.

25. Albert, V., and Hall, M.N. (2015). mTOR signaling in cellular and organismal energetics. Curr. Opin. Cell Biol.

26. Laplante, M., and Sabatini, D.M. (2012). mTOR Signaling in Growth Control and Disease. Cell 149, 274-293.

27. Sancak, Y., Peterson, T.R., Shaul, Y.D., Lindquist, R.A., Thoreen, C.C., Bar-Peled, L., and Sabatini, D.M. (2008). The Rag GTPases Bind Raptor and Mediate Amino Acid Signaling to mTORC1. Science (80-. ). 320.

28. Liu, G.Y., and Sabatini, D.M. (2020). mTOR at the nexus of nutrition, growth, ageing and disease. Nat. Rev. Mol. Cell Biol. 21.

29. Heitman, J., Movva, N.R., and Hall, M.N. (1991). Targets for cell cycle arrest by the immunosuppressant rapamycin in yeast. Science (80-. ).

30. Hatakeyama, R., Péli-Gulli, M.-P., Hu, Z., Jaquenoud, M., Garcia Osuna, G.M., Sardu, A., Dengjel, J., and De Virgilio, C. (2019). Spatially Distinct Pools of TORC1 Balance Protein Homeostasis. Mol. Cell 73, 325-338.e8.

31. Betz, C., and Hall, M.N. (2013). Where is mTOR and what is it doing there? J. Cell Biol. 
$203,563-574$.

32. Tanigawa, M., Yamamoto, K., Nagatoishi, S., Nagata, K., Noshiro, D., Noda, N.N., Tsumoto, K., and Maeda, T. (2021). A glutamine sensor that directly activates TORC1. Commun. Biol. 4, 1093.

33. Nicastro, R., Sardu, A., Panchaud, N., and Virgilio, C. De (2017). The Architecture of the Rag GTPase Signaling Network. Biomolecules 7, 48.

34. Wedaman, K.P., Reinke, A., Anderson, S., Yates, J., McCaffery, J.M., and Powers, T. (2003). Tor Kinases Are in Distinct Membrane-associated Protein Complexes in Saccharomyces cerevisiae. Mol. Biol. Cell 14.

35. Sancak, Y., Bar-Peled, L., Zoncu, R., Markhard, A.L., Nada, S., and Sabatini, D.M. (2010). Ragulator-Rag Complex Targets mTORC1 to the Lysosomal Surface and Is Necessary for Its Activation by Amino Acids. Cell 141.

36. Binda, M., Péli-Gulli, M.-P., Bonfils, G., Panchaud, N., Urban, J., Sturgill, T.W., Loewith, R., and Virgilio, C. De (2009). The Vam6 GEF Controls TORC1 by Activating the EGO Complex. Mol. Cell 35.

37. Devasahayam, G., Burke, D.J., and Sturgill, T.W. (2007). Golgi Manganese Transport Is Required for Rapamycin Signaling in Saccharomyces cerevisiae. Genetics 177.

38. Devasahayam, G., Ritz, D., Helliwell, S.B., Burke, D.J., and Sturgill, T.W. (2006). Pmr1, a Golgi Ca2+/Mn2+-ATPase, is a regulator of the target of rapamycin (TOR) signaling pathway in yeast. Proc. Natl. Acad. Sci. 103.

39. Kaufman, R.J., Swaroop, M., and Murtha-Riel, P. (1994). Depletion of Manganese within the Secretory Pathway Inhibits O-Linked Glycosylation in Mammalian Cells. Biochemistry 33.

40. Luk, E.E.-C., and Culotta, V.C. (2001). Manganese Superoxide Dismutase in Saccharomyces cerevisiae Acquires Its Metal Co-factor through a Pathway Involving the Nramp Metal Transporter, Smf2p. J. Biol. Chem. 276.

41. Muncanovic, D., Justesen, M.H., Preisler, S.S., and Pedersen, P.A. (2019). Characterization of Hailey-Hailey Disease-mutants in presence and absence of wild type SPCA1 using Saccharomyces cerevisiae as model organism. Sci. Rep. 9.

42. Eltschinger, S., and Loewith, R. (2016). TOR Complexes and the Maintenance of Cellular Homeostasis. Trends Cell Biol. 26, 148-159. 
43. Kanki, T., Wang, K., and Klionsky, D.J. (2010). A genomic screen for yeast mutants defective in mitophagy. Autophagy 6.

44. Kanki, T., Kang, D., and Klionsky, D.J. (2009). Monitoring mitophagy in yeast: The Om45-GFP processing assay. Autophagy 5, 1186-1189.

45. Ruiz-Roig, C., Noriega, N., Duch, A., Posas, F., and de Nadal, E. (2012). The Hog1 SAPK controls the Rtg1/Rtg3 transcriptional complex activity by multiple regulatory mechanisms. Mol. Biol. Cell 23.

46. Alarcon, C.M., Heitman, J., and Cardenas, M.E. (1999). Protein kinase activity and identification of a toxic effector domain of the target of rapamycin TOR proteins in yeast. Mol. Biol. Cell 10, 2531-2546.

47. Nicastro, R., Raucci, S., Michel, A.H., Stumpe, M., Osuna, G.M.G., Jaquenoud, M., Kornmann, B., and Virgilio, C. De (2021). Indole-3-acetic acid is a physiological inhibitor of TORC1 in yeast. PLOS Genet. 17.

48. McNaughton, R.L., Reddi, A.R., Clement, M.H.S., Sharma, A., Barnese, K., Rosenfeld, L., Gralla, E.B., Valentine, J.S., Culotta, V.C., and Hoffman, B.M. (2010). Probing in vivo Mn2+ speciation and oxidative stress resistance in yeast cells with electron-nuclear double resonance spectroscopy. Proc. Natl. Acad. Sci. 107, 15335-15339.

49. Urban, J., Soulard, A., Huber, A., Lippman, S., Mukhopadhyay, D., Deloche, O., Wanke, V., Anrather, D., Ammerer, G., Riezman, H., et al. (2007). Sch9 Is a Major Target of TORC1 in Saccharomyces cerevisiae. Mol. Cell 26, 663-674.

50. Hatakeyama, R. (2021). Pib2 as an Emerging Master Regulator of Yeast TORC1. Biomolecules 11, 1489.

51. Chen, Z., Malia, P.C., Hatakeyama, R., Nicastro, R., Hu, Z., Péli-Gulli, M.-P., Gao, J., Nishimura, T., Eskes, E., Stefan, C.J., et al. (2021). TORC1 Determines Fab1 Lipid Kinase Function at Signaling Endosomes and Vacuoles. Curr. Biol. 31.

52. Bodineau, C., Tomé, M., Courtois, S., Costa, A.S.H., Sciacovelli, M., Rousseau, B., Richard, E., Vacher, P., Parejo-Pérez, C., Bessede, E., et al. (2021). Two parallel pathways connect glutamine metabolism and mTORC1 activity to regulate glutamoptosis. Nat. Commun. 12.

53. Bryan, M.R., Uhouse, M.A., Nordham, K.D., Joshi, P., Rose, D.I.R., O’Brien, M.T., Aschner, M., and Bowman, A.B. (2018). Phosphatidylinositol 3 kinase (PI3K) modulates 
manganese homeostasis and manganese-induced cell signaling in a murine striatal cell line. Neurotoxicology 64, 185-194.

54. Noda, T., and Ohsumi, Y. (1998). Tor, a Phosphatidylinositol Kinase Homologue, Controls Autophagy in Yeast. J. Biol. Chem. 273.

55. Villar, V.H., Nguyen, T.L., Delcroix, V., Terés, S., Bouchecareilh, M., Salin, B., Bodineau, C., Vacher, P., Priault, M., Soubeyran, P., et al. (2017). mTORC1 inhibition in cancer cells protects from glutaminolysis-mediated apoptosis during nutrient limitation. Nat. Commun. 8.

56. Klionsky, D.J., Abdelmohsen, K., Abe, A., Abedin, M.J., Abeliovich, H., Arozena, A.A., Adachi, H., Adams, C.M., Adams, P.D., Adeli, K., et al. (2016). Guidelines for the use and interpretation of assays for monitoring autophagy (3rd edition). Autophagy 12.

57. Nevo, Y., and Nelson, N. (2006). The NRAMP family of metal-ion transporters. Biochim. Biophys. Acta - Mol. Cell Res. 1763.

58. Jensen, L.T., Carroll, M.C., Hall, M.D., Harvey, C.J., Beese, S.E., and Culotta, V.C. (2009). Down-Regulation of a Manganese Transporter in the Face of Metal Toxicity. Mol. Biol. Cell 20.

59. Jewell, J.L., Kim, Y.C., Russell, R.C., Yu, F.-X., Park, H.W., Plouffe, S.W., Tagliabracci, V.S., and Guan, K.-L. (2015). Differential regulation of mTORC1 by leucine and glutamine. Science (80-. ). 347, 194-198.

60. Zhang, Z., Yan, J., Bowman, A.B., Bryan, M.R., Singh, R., and Aschner, M. (2020). Dysregulation of TFEB contributes to manganese-induced autophagic failure and mitochondrial dysfunction in astrocytes. Autophagy 16.

61. Settembre, C., Zoncu, R., Medina, D.L., Vetrini, F., Erdin, S., Erdin, S., Huynh, T., Ferron, M., Karsenty, G., Vellard, M.C., et al. (2012). A lysosome-to-nucleus signalling mechanism senses and regulates the lysosome via mTOR and TFEB. EMBO J. 31, 1095-1108.

62. Siddiqui, A., Bhaumik, D., Chinta, S.J., Rane, A., Rajagopalan, S., Lieu, C.A., Lithgow, G.J., and Andersen, J.K. (2015). Mitochondrial Quality Control via the PGC1 -TFEB Signaling Pathway Is Compromised by Parkin Q311X Mutation But Independently Restored by Rapamycin. J. Neurosci. 35.

63. Wang, C., Guan, Y., Lv, M., Zhang, R., Guo, Z., Wei, X., Du, X., Yang, J., Li, T., Wan, Y., et al. 
(2018). Manganese Increases the Sensitivity of the cGAS-STING Pathway for DoubleStranded DNA and Is Required for the Host Defense against DNA Viruses. Immunity 48.

64. Lv, M., Chen, M., Zhang, R., Zhang, W., Wang, C., Zhang, Y., Wei, X., Guan, Y., Liu, J., Feng, K., et al. (2020). Manganese is critical for antitumor immune responses via cGAS-STING and improves the efficacy of clinical immunotherapy. Cell Res. 30.

65. Mossmann, D., Park, S., and Hall, M.N. (2018). mTOR signalling and cellular metabolism are mutual determinants in cancer. Nat. Rev. Cancer 18, 744-757.

66. Mohr, M.R., Erdag, G., Shada, A.L., Williams, M.E., Slingluff, C.L., and Patterson, J.W. (2011). Two Patients With Hailey-Hailey Disease, Multiple Primary Melanomas, and Other Cancers. Arch. Dermatol. 147, 211.

67. García-Rodríguez, N., del Carmen Díaz de la Loza, M., Andreson, B., Monje-Casas, F., Rothstein, R., and Wellinger, R.E. (2012). Impaired Manganese Metabolism Causes Mitotic Misregulation. J. Biol. Chem. 287.

68. Cialfi, S., Pera, L. Le, Blasio, C. De, Mariano, G., Palermo, R., Zonfrilli, A., Uccelletti, D., Palleschi, C., Biolcati, G., Barbieri, L., et al. (2016). The loss of ATP2C1 impairs the DNA damage response and induces altered skin homeostasis: Consequences for epidermal biology in Hailey-Hailey disease. Sci. Rep. 6.

69. Racette, B.A., McGee-Minnich, L., Moerlein, S.M., Mink, J.W., Videen, T.O., and Perlmutter, J.S. (2001). Welding-related parkinsonism. Neurology 56.

70. Santini, E., Heiman, M., Greengard, P., Valjent, E., and Fisone, G. (2009). Inhibition of mTOR Signaling in Parkinson's Disease Prevents L-DOPA-Induced Dyskinesia. Sci. Signal. 2 .

71. Cheng, J., Liao, Y., Dong, Y., Hu, H., Yang, N., Kong, X., Li, S., Li, X., Guo, J., Qin, L., et al. (2020). Microglial autophagy defect causes parkinson disease-like symptoms by accelerating inflammasome activation in mice. Autophagy 16.

72. Ravikumar, B., Vacher, C., Berger, Z., Davies, J.E., Luo, S., Oroz, L.G., Scaravilli, F., Easton, D.F., Duden, R., O’Kane, C.J., et al. (2004). Inhibition of mTOR induces autophagy and reduces toxicity of polyglutamine expansions in fly and mouse models of Huntington disease. Nat. Genet. 36.

73. McColgan, P., and Tabrizi, S.J. (2018). Huntington's disease: a clinical review. Eur. J. 
Neurol. 25.

74. MACDONALD, M. (1993). A novel gene containing a trinucleotide repeat that is expanded and unstable on Huntington's disease chromosomes. Cell 72.

75. Bowman, A.B., Kwakye, G.F., Hernández, E.H., and Aschner, M. (2011). Role of manganese in neurodegenerative diseases. J. Trace Elem. Med. Biol. 25.

76. Chakrabortee, S., Byers, J.S., Jones, S., Garcia, D.M., Bhullar, B., Chang, A., She, R., Lee, L., Fremin, B., Lindquist, S., et al. (2016). Intrinsically Disordered Proteins Drive Emergence and Inheritance of Biological Traits. Cell 167, 369-381.e12.

77. Hesketh, S., Sassoon, J., Knight, R., and Brown, D.R. (2008). Elevated manganese levels in blood and CNS in human prion disease. Mol. Cell. Neurosci. 37.

78. Péli-Gulli, M.-P., Sardu, A., Panchaud, N., Raucci, S., and Virgilio, C. De (2015). Amino Acids Stimulate TORC1 through Lst4-Lst7, a GTPase-Activating Protein Complex for the Rag Family GTPase Gtr2. Cell Rep. 13. 


\section{Figure legends}

Figure 1: NRAMP transporters link Mn-import to rapamycin resistance. (A) Schematical outline of yeast Mn-transporters and their intracellular localization. PM, plasma membrane; E, endosomes; V, vacuoles; M, mitochondria; ER, endoplasmic reticulum; G, Golgi. Note that Bsd2 is a specific adaptor protein for Rsp5-mediated Smf1 and Smf2 ubiquitination. The Golgi Mn-transporter Gdt1 is omitted for clarity. (B-D) Growth on $\mathrm{MnCl}_{2}$ - and/or rapamycin- (RAP) containing medium. 10-fold dilutions of exponentially growing cells are shown. Strains and compound concentrations are indicated. Note that the medium used in (D) was supplemented with $10 \mathrm{mM} \mathrm{CaCl}_{2}$. Data obtained in medium without $\mathrm{CaCl}_{2}$ are shown in Supplementary Figure S3. (E) Southern blot analysis of telomere length. Genomic DNA was derived from cells grown in $\mathrm{CaCl}_{2}$ or $\mathrm{MnCl}_{2}$ containing medium and cleaved by XhoI before agarose gel electrophoresis. The $1.3 \mathrm{~kb}$ average length of telomeres from wild-type cells (dashed white line, black arrow) and size marker (M) are shown. (F) Growth of pmr1 $1 \Delta m f 2 \Delta$ double mutants transformed with plasmids expressing yeast Smf2, Mus musculus Slc11a1, or Slc11a2 on RAP-containing medium.

Figure 2: Rapamycin and intracellular Mn detoxification affect Smf1 and Smf2 protein turnover. (A) Exponentially growing wild-type (WT), pmr1 $1 \Delta$ and $b s d 2 \Delta$ cells expressing a GFP-Smf1 fusion protein from plasmid pRS416-pSMF1-GFP-SMF1 were treated with 200 $\mathrm{ng} / \mathrm{ml}$ rapamycin (RAP) for up to 2 hrs. GFP-Smf1 and cleaved GFP (*GFP) protein levels were analyzed by immunoblotting using an anti-GFP antibody. G-6-PDH protein levels were used as loading control. (B) Quantification of GFP-Smf1 and cleaved GFP normalized to corresponding untreated cells are shown. Data represent means \pm SEM of independent experiments $(n=3)$. Statistical analysis: paired two-tailed t-test. ${ }^{*} \mathrm{p}<0,05$; $* * \mathrm{p}<0,01$. (C) Quantification of GFP-Smf1 and cleaved GFP in untreated cells normalized to the wild-type are shown. Other details as in (B). ${ }^{* * *} \mathrm{p}<0,001$. (D) Microscopic analysis of GFP-Smf1 localization in exponentially growing wild-type (WT), $p m r 1 \Delta$ and $b s d 2 \Delta$ cells expressing a GFP-Smf1 fusion protein from plasmid pRS416-pSMF1-GFP-SMF1 upon treatment with 200 ng/ml rapamycin (RAP) for 2 hrs. Scale bar represents $5 \mu \mathrm{m}$. (E-G) Exponentially growing wild-type (WT), pmr1 $\Delta$ and $b s d 2 \Delta$ cells expressing a Smf2-GFP fusion protein from its endogenous locus were treated with $200 \mathrm{ng} / \mathrm{ml}$ rapamycin (RAP) for up to $2 \mathrm{hrs}$. Smf2-GFP 
and cleaved GFP $\left({ }^{*} \mathrm{GFP}\right)$ protein levels were analyzed by immunoblotting with an anti-GFP antibody. Quantifications are shown in (F) and (G). Details as described in (B) and (C). (F) Microscopic analysis of Smf2-GFP localization in exponentially growing wild-type (WT),

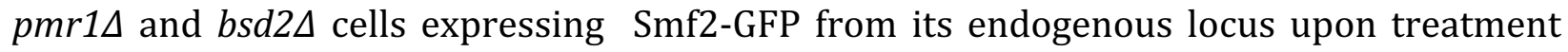
with $200 \mathrm{ng} / \mathrm{ml}$ rapamycin (RAP) for $2 \mathrm{hrs}$. Scale bar represents $5 \mu \mathrm{m}$.

Figure 3: Intracellular $\mathrm{Mn}$ excess interferes with rapamycin-induced autophagy, mitophagy and Rtg3 activation. (A) Exponentially growing cells transformed with plasmid VGp160 were treated for up to $6 \mathrm{hrs}$ with $200 \mathrm{ng} / \mathrm{ml}$ rapamycin (RAP). GFP-Atg8 and cleaved GFP $(*$ GFP) protein levels were analyzed by immunoblotting. G-6-PDH protein levels were used as loading control. (B) Quantification of cleaved GFP at 6 hrs normalized to the wild type is shown. Data represent means \pm SEM of independent experiments $(n=3)$. Statistical analysis: paired two-tailed t-test. * $\mathrm{p}<0,05$; ** $\mathrm{p}<0,01$; *** $\mathrm{p}<0,001$. (C) Exponentially growing cells expressing Om45-GFP from the endogenous locus were treated for up to $6 \mathrm{hrs}$ with $200 \mathrm{ng} / \mathrm{ml}$ rapamycin (RAP). Om45-GFP and cleaved GFP (*GFP) protein levels were analyzed by immunoblotting. Other details as in (A). (D) Quantification of cleaved GFP at $6 \mathrm{hrs}$ normalized to the wild type is shown. Other details as in (B). (E)

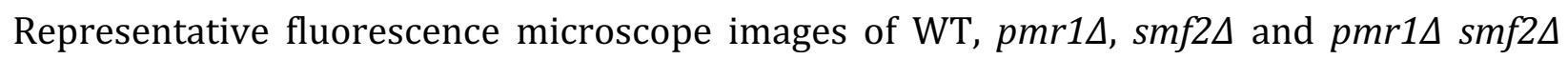
mutants expressing an episomic Rtg3-GFP reporter construct. Exponentially growing cells were treated for 30 minutes with $200 \mathrm{ng} / \mathrm{ml}$ rapamycin (+RAP). Scale bar represents $5 \mu \mathrm{m}$. (F) Exponentially growing cells transformed with plasmid pRS416-RTG3 were treated for up to 30 minutes with $200 \mathrm{ng} / \mathrm{ml}$ rapamycin (RAP). Rtg3-GFP protein levels were analyzed by immunoblotting. G-6-PDH protein levels were used as loading control.

Figure 4: $\mathrm{MnCl}_{2}$ stimulates TORC1 kinase activity in vitro and in vivo. (A) In vitro TORC1 kinase assays using $\left[\gamma^{-32} \mathrm{P}\right]-\mathrm{ATP}$, recombinant Lst4Loop as substrate, and increasing concentrations (2-fold dilutions) of $\mathrm{MgCl}_{2}$ or $\mathrm{MnCl}_{2}$. Substrate phosphorylation was detected by autoradiography (left) and Sypro Ruby staining is shown as loading control (right). (B) Quantification of the assay shown in (A). Curve fitting and parameter calculations were performed with GraphPad Prism. Data shown are means $( \pm$ SEM, $n=3)$. (C) In vivo TORC1 activity in exponentially growing cells treated (+) or not (-) with $\mathrm{MnCl}_{2}$ 
for 1h. TORC1 activity was assessed by immunoblotting using phosphospecific antibodies against the bona fide TORC1 target residue $\mathrm{Thr}^{737}$ in Sch9 and anti-Sch9 antibodies. (D). Rapamycin-resistant growth of pmr1s cells requires the presence of the Rag GTPase Gtr1. Cells with the indicated genotypes, harboring a control plasmid ( $\mathrm{p} E M P T Y$ ) or a plasmid that allows expression of Gtr1 (pGTR1) were 10-fold serially diluted, spotted on plates without (control) or with $4 \mathrm{ng} / \mathrm{ml}$ rapamycin (RAP), and grown for 3 days at $30^{\circ} \mathrm{C}$.

Figure 5: $\mathrm{MnCl}_{2}$ activates mTORC1 activation signaling in human cells. (A-B) Human U2OS (A) and HEK293T (B) cells were starved for all amino acids (-AA) and supplemented with increasing amounts of $\mathrm{MnCl}_{2}$ as indicated for 2 hours. Phosphorylation of the mTORC1 downstream targets S6K and S6 was assessed by immunoblot analysis. A control with cells incubated in the presence of all proteinogenic amino acids (+AA) was included as a positive control. (C) Human U2OS were starved for all amino acids (-AA) and supplemented with $0.35 \mathrm{mM}$ of $\mathrm{MnCl}_{2}$ for 2 hours. Phosphorylation of S6K, S6, 4EBP1, ULK1 and AKT was assessed by immunoblot analysis. A control with cells incubated in the presence of all proteinogenic amino acids ( + AA) was included as a positive control. (D-E) Human U20S cells were treated as in $\mathrm{C}$ for $4 \mathrm{~h}$ either in the absence (D) or the presence (E) of chloroquine (CQ). Autophagic marker LC3I/II was then analyzed by immunoblot. (F-G) GFP-LC3 expressing U20S cells were incubated as in D. GFP-LC3 aggregation was assessed by confocal microscopy (F), and quantified using ImageJ software (G). Scale bar indicates 10 $\mu \mathrm{m}$. Values were normalized to $-\mathrm{AA}$ condition. Graphs represent average \pm S.D., with $\mathrm{n}=3$ (*, $\mathrm{p}<0.05$, ANOVA followed by Turkey's test). (H) Model of Mn-driven TORC1 activation. NRAMP-dependent increase in cytoplasmatic Mn is further enhanced by impaired Mn import into Golgi. Mn activated TORC1 is then able to cope with rapamycin-mediated TORC1 inhibition (see rosa and blue boxes). 
bioRxiv preprint doi: https://doi.org/10.1101/2021.12.09.471923; this version posted December 9, 2021. The copyright holder for this preprint (which was not certified by peer review) is the author/funder. All rights reserved. No reuse allowed without permission.

A

$\mathrm{Mn}^{2}$

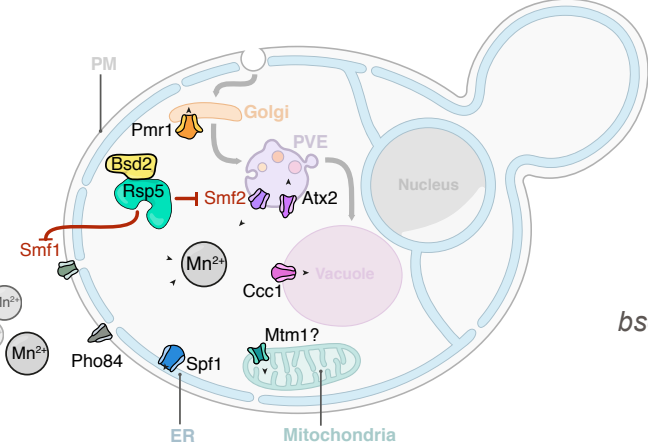

B

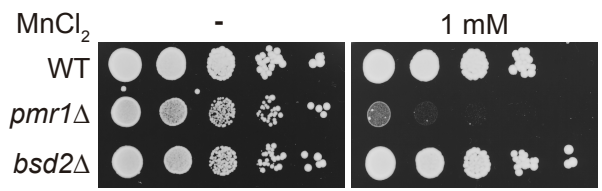

D

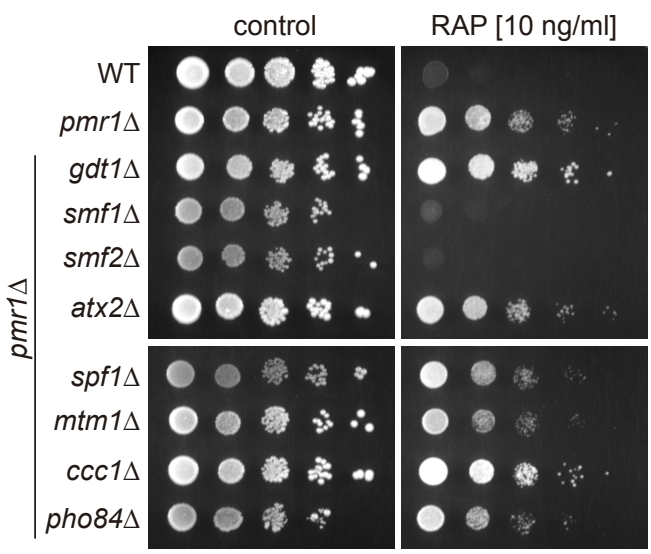

F

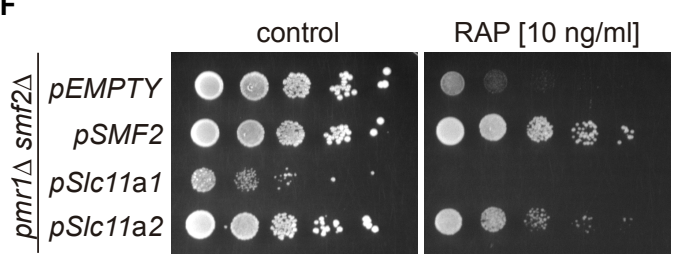

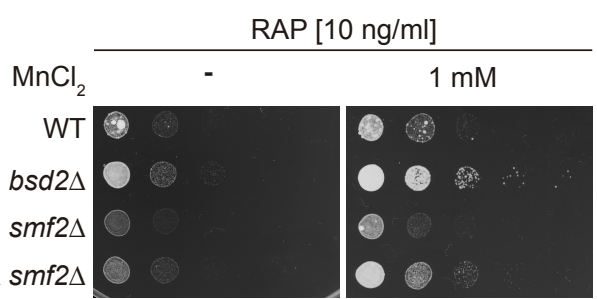

RAP [10 ng/ml]

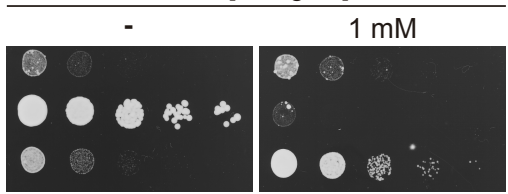

E

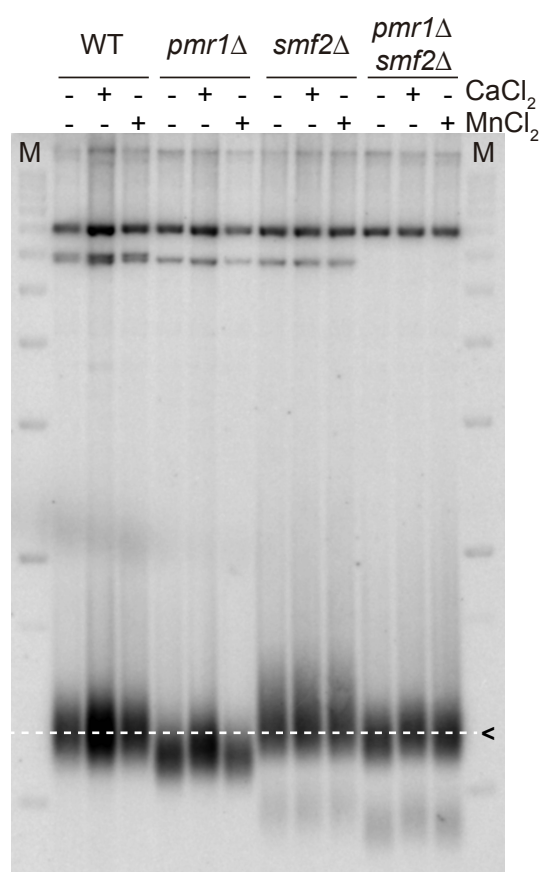

Figure 1 
A

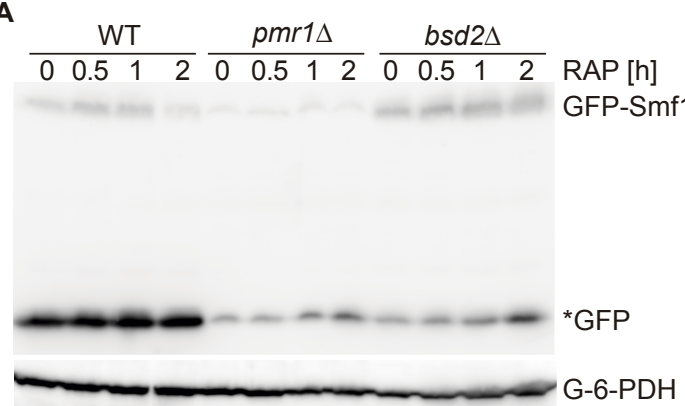

C
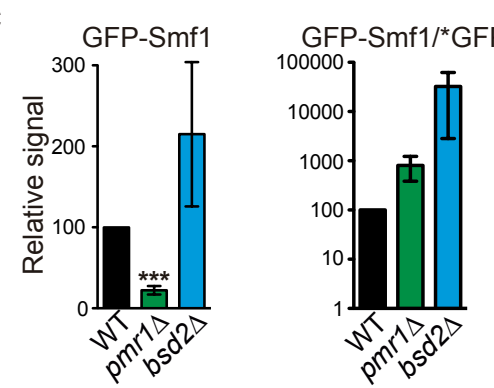

E

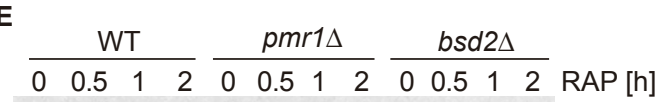

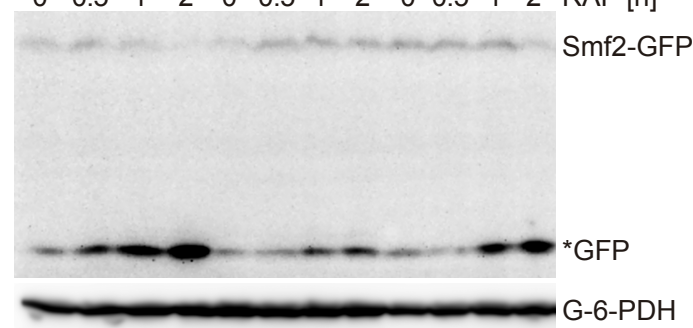

G

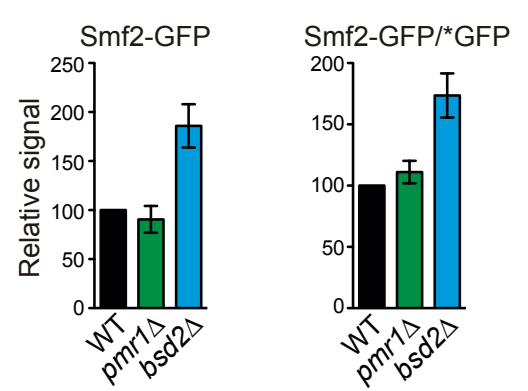

B

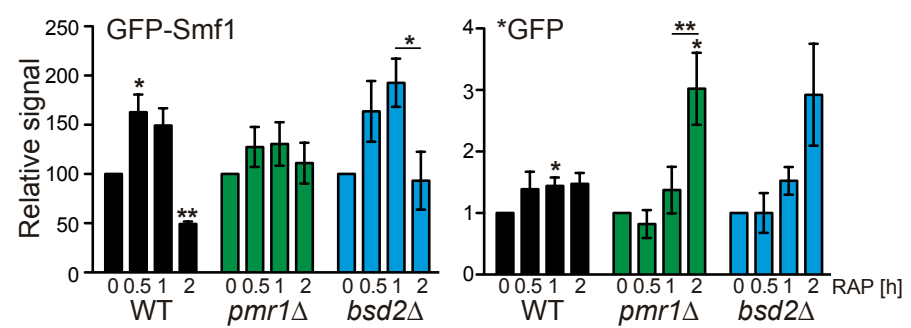

D

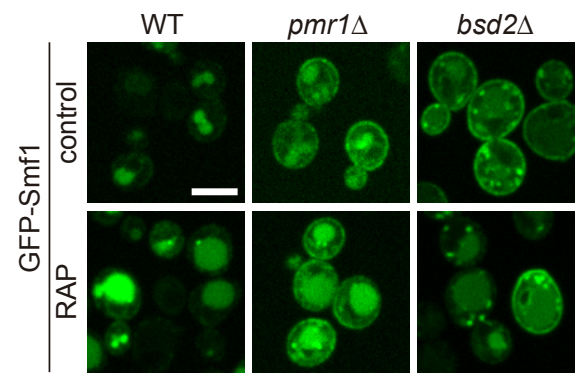

F

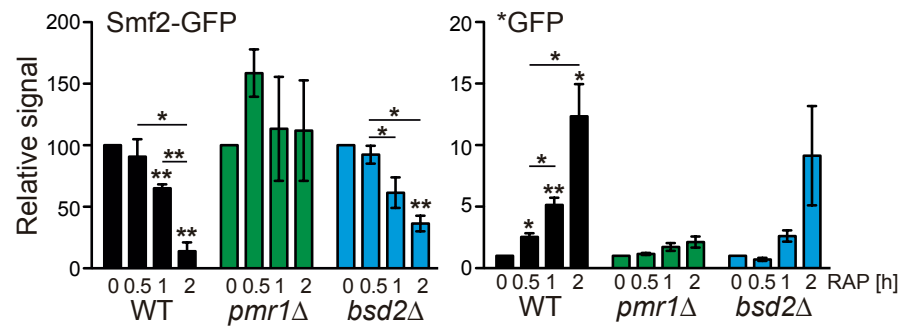

H

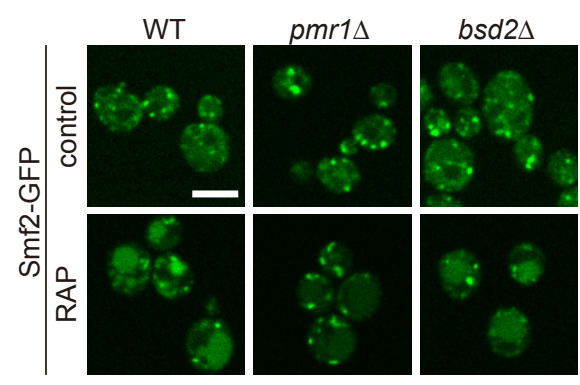

Figure 2 
bioRxiv preprint doi: https://doi.org/10.1101/2021.12.09.471923; this version posted December 9, 2021. The copyright holder for this preprint (which was not certified by peer review) is the author/funder. All rights reserved. No reuse allowed without permission.

A

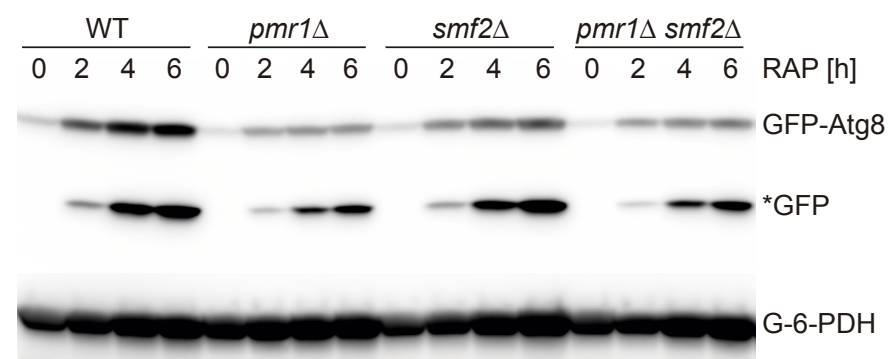

C

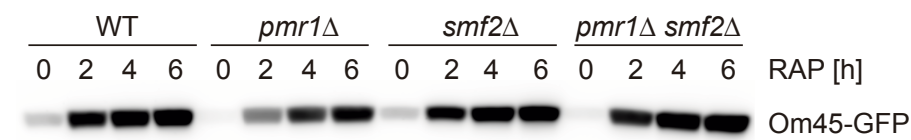

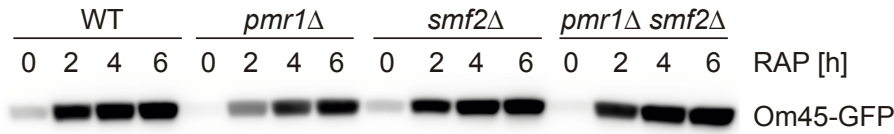

- *GFP

G-6-PDH
B
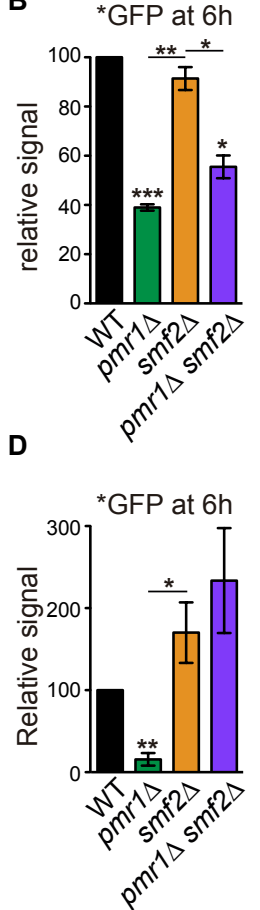

E

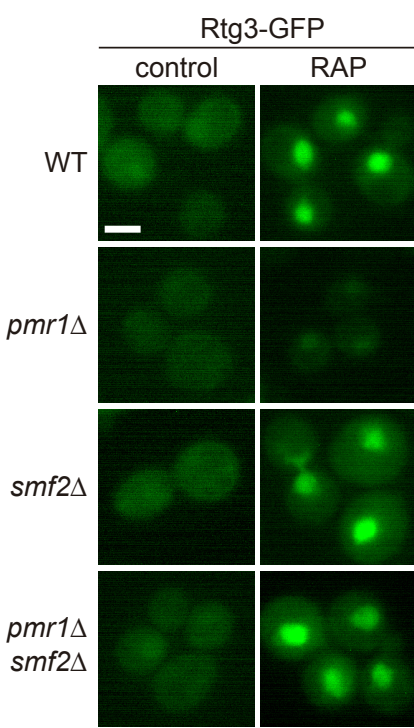

\begin{tabular}{|c|c|c|c|c|c|}
\hline & WT & $p m r 1 \Delta$ & $s m f 2 \Delta$ & $\begin{array}{l}p m r 1 \Delta \\
s m f 2 \Delta\end{array}$ & \\
\hline 0 & 1530 & $0 \quad 1530$ & $\begin{array}{lll}0 & 1530\end{array}$ & $0 \quad 1530$ & $\mathrm{RAP}$ [min] \\
\hline
\end{tabular}

Figure 3 
bioRxiv preprint doi: https://doi.org/10.1101/2021.12.09.471923; this version posted December 9, 2021. The copyright holder for this preprint (which was not certified by peer review) is the author/funder. All rights reserved. No reuse allowed without permission.

A

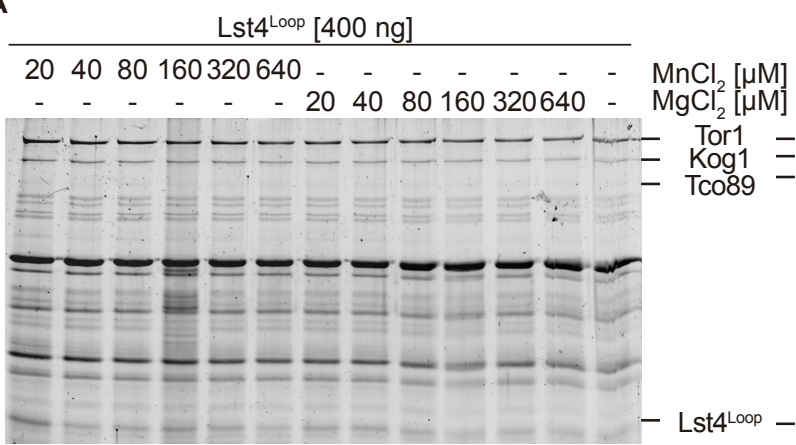
Lst4 ${ }^{\text {Loop }}[400 \mathrm{ng}$ ]

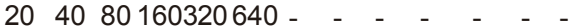
- - - - - - 204080160320640 -
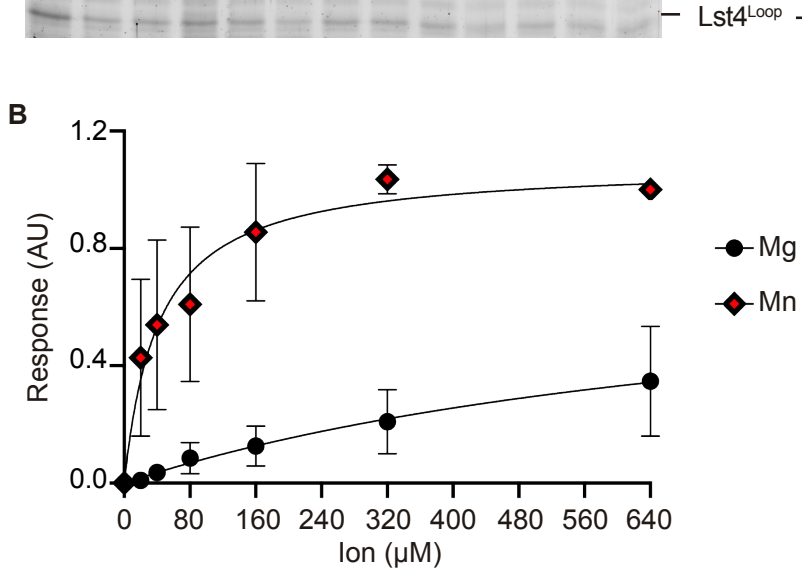

C

D

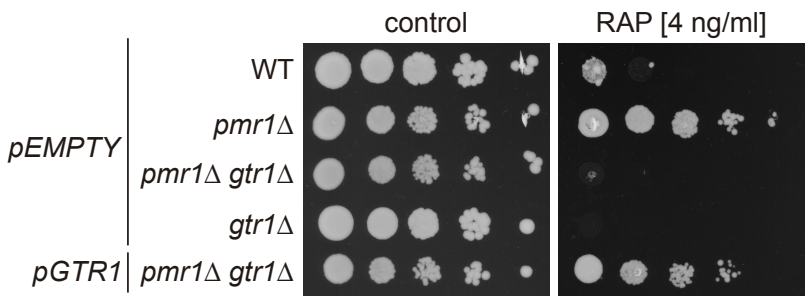

Figure 4 
bioRxiv preprint doi: https://doi.org/10.1101/2021.12.09.471923; this version posted December 9, 2021. The copyright holder for this preprint (which was not certified by peer review) is the author/funder. All rights reserved. No reuse allowed without permission.

A

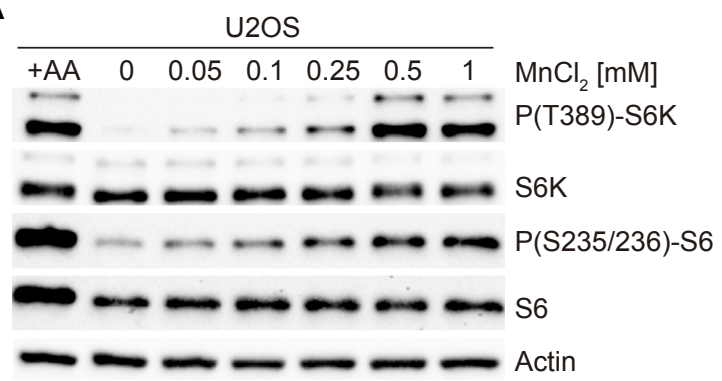

B

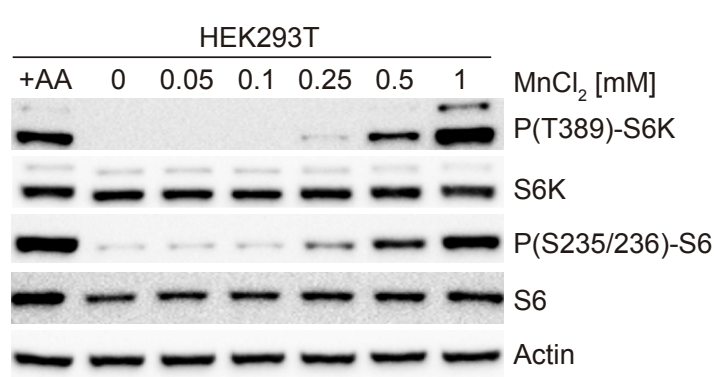

$\mathbf{E}$

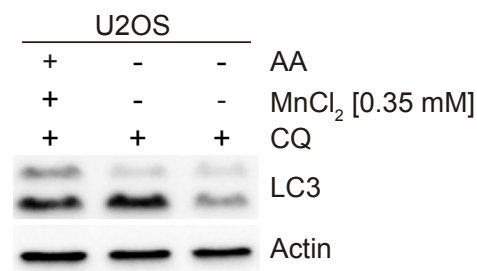

$\mathbf{F}$ U2OS GFP-LC3 (4h)

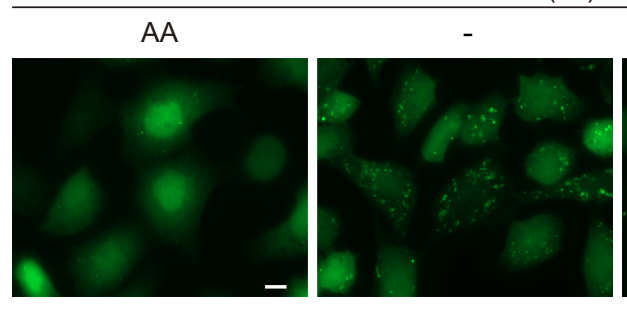
$\mathrm{MnCl}_{2}[0.35 \mathrm{mM}]$

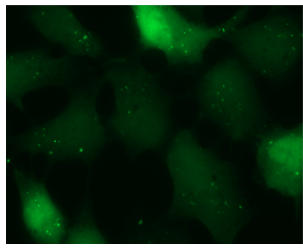

H

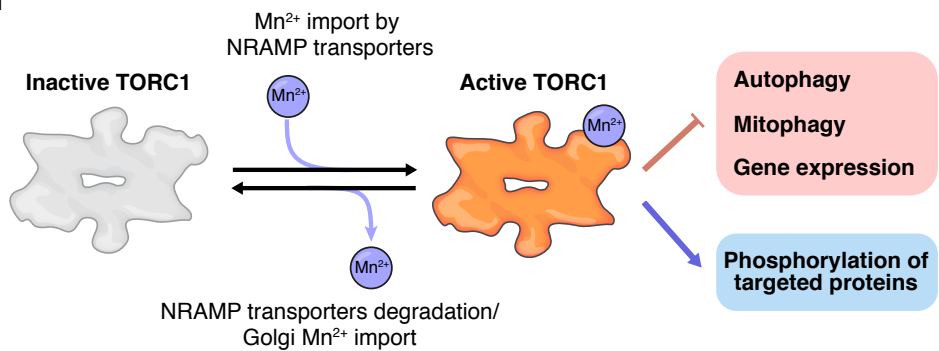

Figure 5 